\title{
Mechanical Characterization of Mesoscale Interfaces Using Indentation Techniques
}

\author{
SURYA R. KALIDINDI, ${ }^{1,3}$ SOUMYA MOHAN,${ }^{1}$ and ALICIA ROSSI ${ }^{2}$ \\ 1.-Woodruff School of Mechanical Engineering, Georgia Institute of Technology, Atlanta GA, \\ USA. 2.-School of Material Science and Engineering, Georgia Institute of Technology, Atlanta, \\ GA, USA. 3.—e-mail: surya.kalidindi@me.gatech.edu
}

\begin{abstract}
Mesoscale interfaces and interphases play a central role in controlling the many macroscale mechanical properties and performance characteristics of structural materials. Modern instrumented indenters present an unprecedented opportunity to measure, reliably and consistently, the local mechanical responses at a multitude of length scales ranging from tens of nanometers to hundreds of microns. When these high-fidelity measurements are combined with rigorous data analyses protocols, it is possible to systematically study the mechanical role of individual mesoscale interfaces and quantify their contributions to the overall mechanical response of the material system. The advantages of these new measurement and analyses protocols as well as the potential for development and implementation of novel high-throughput assays is discussed.
\end{abstract}

\section{INTRODUCTION}

Since most advanced materials of interest in emerging technologies exhibit rich heterogeneity at a multitude of hierarchical length/structure scales (spanning the atomistic to the macroscale), it should not be surprising that these materials abound with a variety of interfaces (or interphases). ${ }^{1,2}$ Furthermore, it is well known and understood that these interfaces play a dominant role in controlling many of the failure-related mechanical properties of the material (e.g., Refs. $3-8)$. Interestingly, these effects have included both beneficial (e.g., strengthening, toughening) and detrimental (e.g., embrittlement) contributions, often pushing the effective properties outside the range of the properties of the constituents.

In metals and alloys, grain boundaries and phase boundaries are well known for their leading role in phenomena such as creep, ${ }^{9-11}$ grain boundary embrittlement, ${ }^{12-14}$ toughness,${ }^{15}$ and corrosion. ${ }^{16-18}$ However, there do not exist broadly validated models that specifically account for their role. For example, it is a general practice to model the role of grain boundaries in increasing the plastic flow strength in metals using the Hall-Petch laws. ${ }^{19,20}$ However, the most commonly used formulations of the Hall-Petch effect treat all grain boundaries as the same, i.e., they do not discriminate between the different types of grain boundaries in modeling their role in spite of the large body of evidence. ${ }^{21-24} \mathrm{In}$ polymer composites, significant effort has been focused on improving wettability and chemical bonding at or near the interface region. ${ }^{25-32}$ Generally referred as the interphase, this region exhibits a transition between the properties of the matrix and the reinforcement phases. Engineering these interphases for improved macroscale properties presents a significant challenge. Prior efforts have included silane treatments of glass fiber epoxy composites, ${ }^{25,26}$ use of nanotubes to enhance glass fiber-matrix adhesion, ${ }^{28}$ the use of nano-fillers in the matrix ${ }^{33}$ and surface modifications of carbon nanotubes. ${ }^{27}$ Although these studies have clearly demonstrated the enhancement of the bulk mechanical properties, additional work is still needed to understand and quantify the underlying physical processes.

Systematic studies of the mechanical roles of interfaces and interphases necessarily need to start with a rigorous quantification of the salient features describing the material structure associated with them. This is not a trivial task. For example, in order to define the mesoscale degrees of freedom associated with a grain or phase boundary in typical metals, one needs to characterize the grain lattice 
orientations on either side of the boundary as well as the boundary normal, in addition to identifying the thermodynamic phases meeting at the boundary. ${ }^{34}$ Likewise, for interphases in polymer composites, one not only needs to characterize the material chemistry and structure of the adjoining phases at multiple length scales but also their gradients through the interphase. Currently employed techniques for quantifying the mesoscale interfaces and interphases in advanced materials of interest are effort-intensive, destructive, require highly specialized equipment and expertise, and often only produce incomplete information.

Notwithstanding the difficulties already mentioned, the mechanical characterization of mesoscale interfaces and interphases have proven to be even more difficult. One successful approach has involved the use of a focused ion beam (FIB) to extract a 'micro-pillar' of the material including the desired interface, and performing compression tests. ${ }^{35-37}$ However, this technique is not easily scalable to studying the wide variety of distinct interfaces and interphases encountered in advanced material systems of interest. It is also noteworthy that significant advances have been made in studying fiber-matrix interface/interphase strengths using pull-out tests. ${ }^{38,39}$ These tests isolate a single fiber in the matrix. The sample preparation for these tests is tedious, especially when the fibers have exceedingly small diameters, and may not reliably mimic the actual conditions in the real samples. The ability to obtain reliable interface properties from realistic samples is critical to a better understanding of the nature of composite interfaces and their effect on bulk properties.

An alternative that has emerged in recent years which has shown tremendous promise is instrumented indentation. ${ }^{40,41}$ Modern indenters with their impressive load and displacement resolutions and continuous stiffness measurement (CSM) capabilities have now made it possible to study systematically the mechanical responses of regions near mesoscale interfaces as a function of the distance from the interface. While the experiment itself is relatively simple (and capable of high throughput), the central challenges lie in the sample preparation $^{42}$ and in the post-test data analysis protocols employed to recover meaningful properties intrinsic to the material volume probed in the experiment. This review will summarize the main advances already made in studies of mesoscale interfaces in both metals and polymer composites using indentation methods.

\section{INTERFACES AND INTERPHASES IN COMPOSITES}

Indentation techniques used in exploring the mechanical response of interfaces in composites have focused largely on two types of applications. The first application has targeted the transition of mechanical properties across an interphase using nanoindentation. The second application has employed microindentation to quantify fiber debonding and estimate a measure of the interface shear strength. In these applications, the interphase region is formed by bonding and reactions between the reinforcement and the matrix phases. ${ }^{43}$

Figure 1 provides examples of the measurements of the mechanical property transitions in the interphase obtained using a nanoindenter. Figure 1a shows the measured variation of the modulus in the silane-treated interphase region of a polymer-glass fiber composite sample. ${ }^{26}$ It is also clear that one can estimate the thickness of the interphase layer from these measurements. Figure $1 \mathrm{~b}$ shows the measured variation of hardness in the interphase in both unaged (virgin) and aged (in water) conditions. ${ }^{44,45}$ Once again, one can estimate the thickness of the interphase in these samples from these measurements. The hardness measurements obtained in these studies show significantly larger variance compared to the variance in the modulus measurements, some of which may be attributed to the data analyses protocols employed in these studies (these will be discussed in more detail later). The technique has been applied successfully in multiple studies, ${ }^{45-50}$ often in combination with the nanoscratch tests. ${ }^{26,44-47,50}$ The advantage of the nanoscratch tests is that they allow for a more precise identification of the extent of penetration of the interphase region into the matrix. It is generally expected that a smooth gradient in the mechanical properties reduces the stress and/or strain concentrations that occur at the interface, and therefore should have a beneficial impact on the overall strength of the composite.

The trends obtained in the nanoindentation measurements shown in Fig. 1 should largely be treated as qualitative (not quantitative) results. For example, the results clearly confirm the transition in the mechanical properties and the degradation in hardness. However, the significant variance exhibited in the measurements (within the same sample and between different samples) has precluded any reliable quantification of these effects (see Fig. 1).

There are indeed many opportunities for improving the nanoindentation protocols. First, it is important to recognize that the current protocols generally employ sharp indenter tip geometries (e.g., Berkovich indenter ${ }^{40}$ ) and relatively large indentation depths, mainly because the hardness measurements obtained using these protocols provide the most consistent measurements. Indeed, the asymptotic values obtained at the larger indentation depths are often reported as hardness values in these protocols. However, these conditions produce significant plastic strain in the indentation zone and alter the condition of the material being studied. Since the hardness measurements are recovered from the unloading segment of the load-displacement curve (after applying a large amount of plastic 
(a) Vinylester/glass fiber interphase

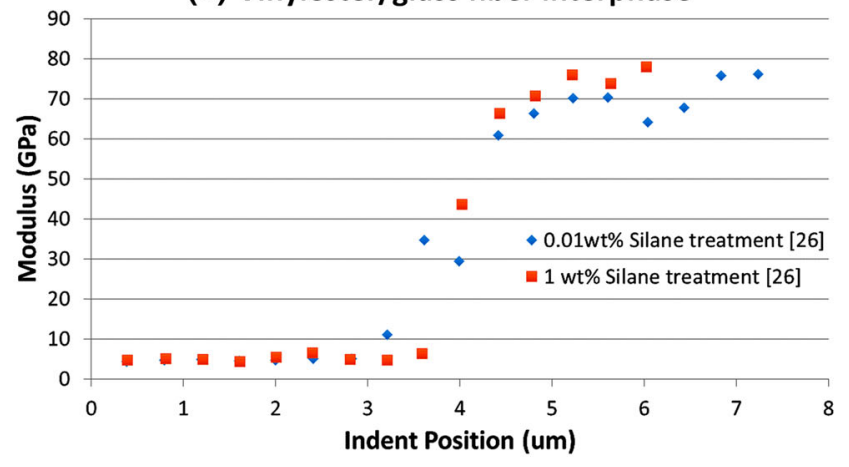

(b) Indentation measurements across interphase

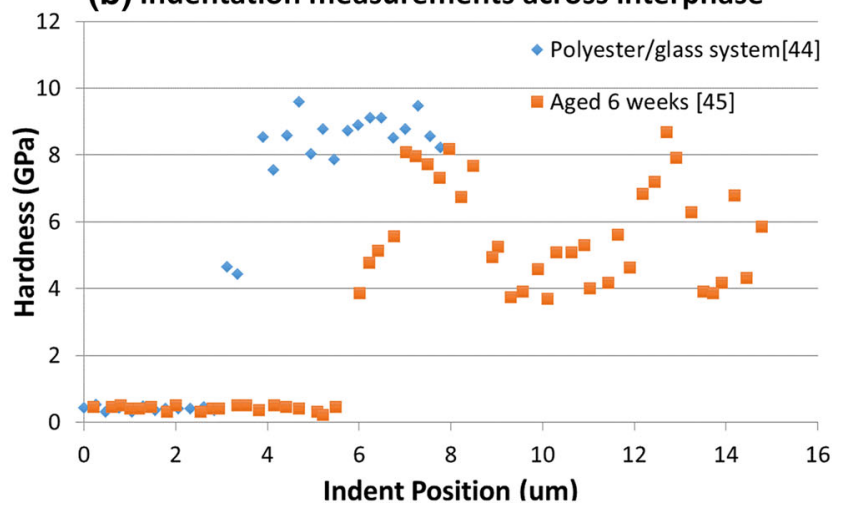

Fig. 1. Sharp indentation measurements on glass fiber composite systems showing the change in properties across the interphase: (a) an interface enhanced with a silane treatment to enhance the bonding between the components(fiber and matrix), ${ }^{26}$ (b) dry and water-aged samples illustrating the change in the properties in the interphase region with aging. ${ }^{44,45}$

deformation), they are indeed affected by the changes induced in the material during the indentation test. Second, since the extracted values of moduli and hardness in the currently employed protocols show substantial sensitivity to sample/ indenter geometries and test conditions (e.g., indentation load and depth levels), the extracted properties from these protocols cannot yet be easily interpreted or related to the intrinsic material properties of interest defined typically in simple (uniaxial) tension or compression tests.

Some progress in the directions mentioned above has been made in recent years with the development and use of spherical indentation stress-strain protocols. ${ }^{41,51-53}$ Most importantly, it has been demonstrated that the use of spherical indenters has the potential to produce indentation stressstrain curves (not just values of modulus and hardness). Furthermore, because the spherical indenters produce significantly lower concentrations in the imposed stress and/or strain fields compared to the sharp indenters, it is possible to control the indentation depths to relatively small values and investigate the local material response at low levels of plastic deformation. Indeed, it is possible to track the indentation response of the material through the initial elastic regime and the subsequent elastic-plastic regime.

The spherical indentation stress-strain protocols are made possible through the use of novel data analyses protocols. ${ }^{54,55}$ These protocols involve rigorous procedures for identifying the initial elastic loading segment in the measured load-displacement curve (as opposed to the unloading segments used in most currently employed protocols). This is accomplished by fitting the load, displacement, and the continuous stiffness measurements from the indentation test to the expected interrelationships between them based on Hertz's theory. ${ }^{56}$ As a result, one can reliably identify an effective zeropoint of initial contact that best fits the raw measurements, and produces a meaningful initial

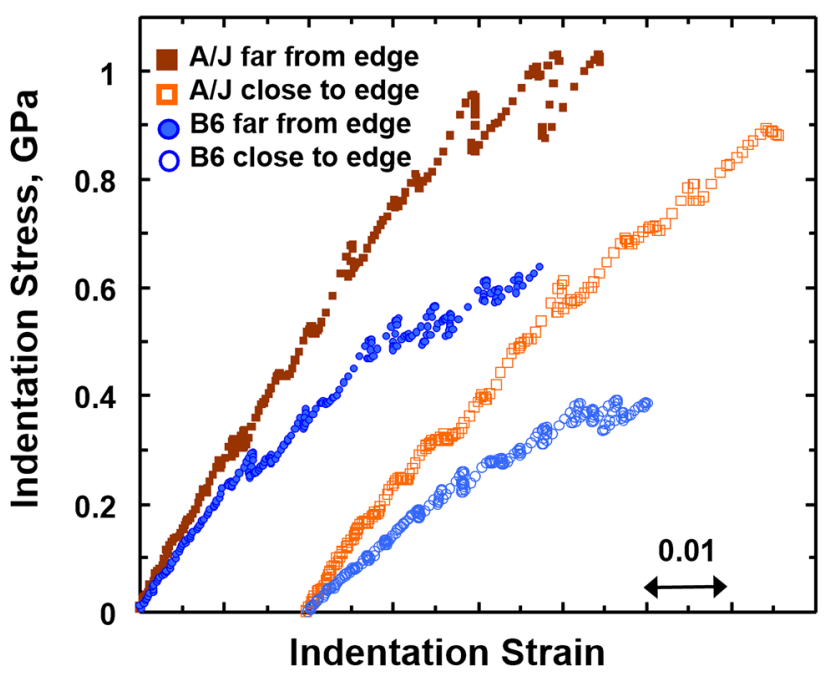

Fig. 2. Indentation stress-strain curves showing the difference in properties in older and newer growth locations in mouse bone. ${ }^{51}$ The raw data were generated from the spherical indentation. An offset is added so that both sets of curves can be seen clearly. $A / J$ and $B 6$ refer to two different inbred mouse strains. Reprinted with permission from Elsevier.

elastic segment of the indentation stress-strain response. This concept of effective initial contact provides a practical way of accounting for various unavoidable factors in the experiments, such as the surface roughness of the sample, small deviations in the indenter tip geometry compared to the idealized spherical geometry and slight misalignment of indentation direction with respect to the sample surface normal, among others. Once the elastic segment is successfully identified, it is relatively easy to extract an indentation modulus, and use it in conjunction with the continuous stiffness measurement to recover the contact radius in the indentation. Contact radius is a central parameter in the definition of both the indentation stress and the indentation strain. 
The novel spherical indentation stress-strain protocols are yet to be applied to polymer composite samples. They have been largely applied thus far to a broad range of metals and alloys, with only a limited number of applications in non-metals, such as brushes of carbon-nanotubes ${ }^{53}$ and bone. ${ }^{51,52}$ These limited applications do provide strong evidence for improving the analyses of the raw data gathered in the indentation measurements and in the extraction of meaningful properties. As a specific example, in the studies on bone, it was demonstrated that it is possible to reliably measure the differences in the local indentation stress-strain curves over very short length scales (of the order of tens of microns) and different mouse strains (see Fig. 2). Furthermore, it was demonstrated that the estimated values of modulus and hardness from these new protocols are significantly more reliable than those extracted from the conventional protocols by correlating the indentation measurements to the measurements of local compositions at the indentation sites obtained using Raman spectroscopy. Herein lies the real promise of these new protocols. When suitably combined with local measurements of material structure at the same (or comparable) length scale of the indentation, these new techniques have the potential to extract reliable structure-property linkages in hierarchical materials in high-throughput assays. This specific capability arises mainly because these new protocols employ relatively short indentation depths leading to fairly small indentation zone sizes (in the range of $50 \mathrm{nms}-50 \mu \mathrm{m}$ ) with only a modest alteration of the material in the indented zone. Although these capabilities have only been firmly established thus far for metal samples (discussed next), these new protocols raise several exciting new prospects for completely new research directions in the study of composite material systems.

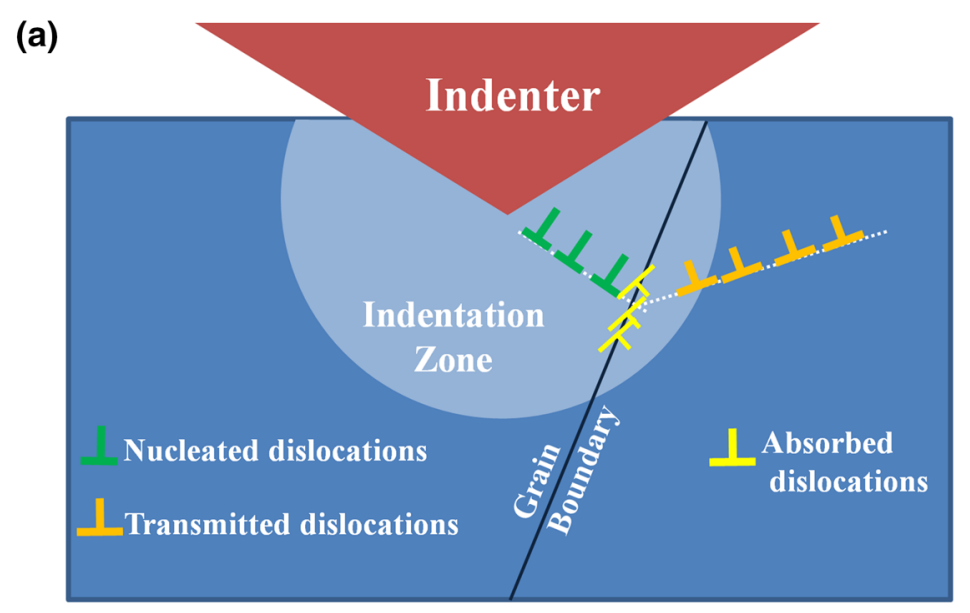

(b)

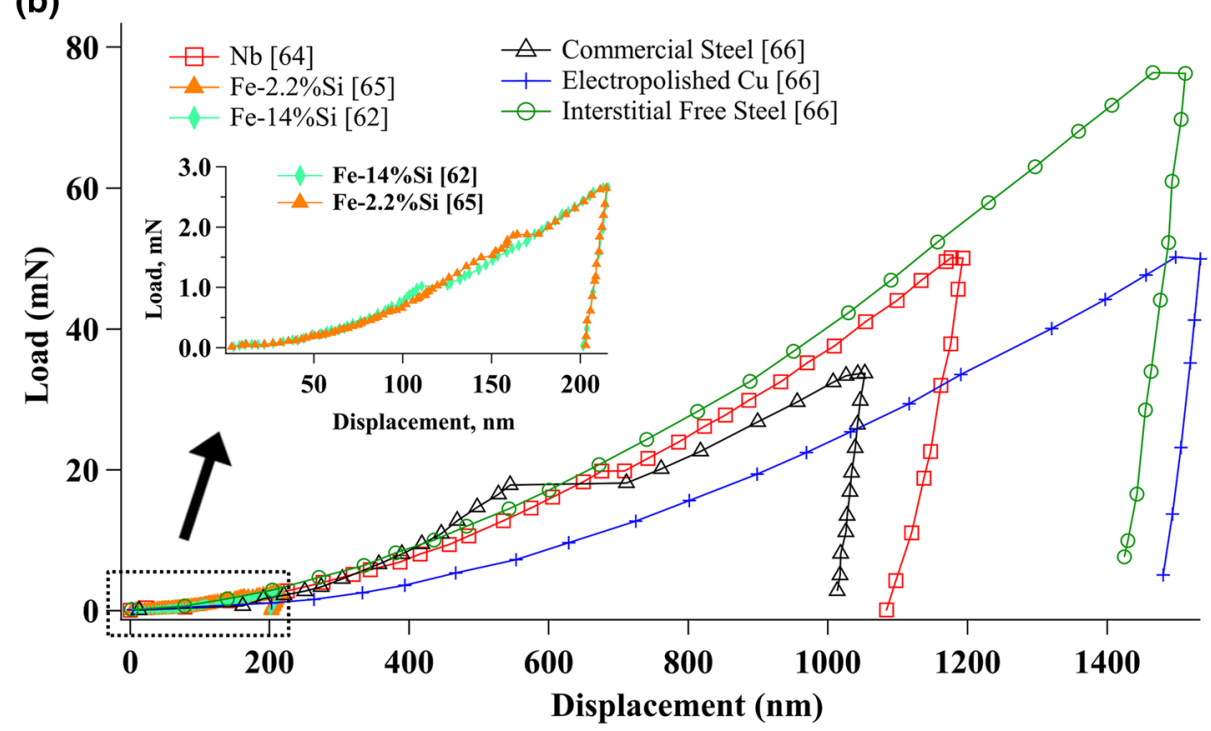

Fig. 3. (a) Schematic of dislocation slip transmission across a grain boundary in the indentation zone. (b) Examples of GB pop-ins observed in previously reported studies. ${ }^{62,64-66}$ Inset shows GB pop-ins at lower loads for Fe-Si-based alloys. ${ }^{62,65}$ 
At the higher length scales, microindentation has been employed to investigate the interfacial shear strength in composite materials. Desaeger and Verpoest ${ }^{57}$ performed indentation experiments approximately at the center of fibers embedded in the matrix. One advantage of this approach is that it can be used on real composite samples instead of an idealized composite sample (e.g., samples with only one fiber imbedded in the matrix). The fibers were indented to various loads, and a determination of debonding was made based on the occurrence of a black halo around the fiber indicating the interface crack. From these results, a debonding load was calculated as the load at which a debonding crack had a $50 \%$ chance of occurring. Interface shear strength was then estimated from the debonding load using the shear lag model. ${ }^{57}$ Microindentation can also be used to measure modulus and hardness at larger length scales using conventional data analyses protocols. ${ }^{58}$ Just as remarked earlier, although these methods have shown tremendous promise in providing new insights, the measurements should be treated as qualitative (i.e., comparative) measures because of the large variance often reflected in such measurements.

\section{GRAIN/PHASE BOUNDARIES IN METALS}

Mechanical characterization of interfaces in metal samples using indentation techniques has been aimed mainly at studying slip transfer at grain boundaries. As with the composites, these studies have mostly employed sharp indenters. The test configuration and the expected deformation processes in the indentation zone are shown schematically in Fig. 3a. As shown in this figure, the dislocations are expected to initially pile-up on the grain boundary, and after overcoming some resistance, they will undergo some combination of absorption at the grain boundary and transmission to the neighboring grain (this might include nucleation and propagation of new dislocations in the neighboring grain). This process should produce a pop-in (sudden jump in displacement in load control indentation tests) in the measured load-displacement curves.

An example of a pop-in that might be attributable to grain boundary resistance is shown in Fig. 3b. It should be noted that pop-ins occur quite regularly in indentation tests on annealed samples, even when the indentations are performed in regions far from the grain boundaries, especially in the tests with the small indenter tips. ${ }^{59,60}$ However, such pop-ins are referred as incipient pop-ins and are generally attributed to the difficulty of instantiating a dislocation source in the very small indentation zone involved in such tests. Interestingly, the incipient pop-ins have been observed to be suppressed or mitigated in indentations conducted close to the grain boundary (compared to those conducted in the grain interior). ${ }^{24,60-63}$ These observations have suggested that the grain boundaries serve as potent sources of dislocations.

In contrast to the incipient pop-ins, the pop-ins shown in Fig. 3b are referred as grain boundary (GB) pop-ins. ${ }^{61,62,64-67}$ These are attributed to slip transfer events at the grain boundary. These occur at significantly higher load levels (after the sample has experienced a significant amount of plasticity in the indentation zone). Wang and $\mathrm{Ngan}^{64}$ correlated the GB pop-ins in a coarse-grained, $99.99 \%$ pure, $\mathrm{Nb}$ sample to a critical $c / d$ value of 2 for a grain boundary with a high degree of alignment between the active slip systems on either side of the grain boundary, where $c$ is the radius of elastic-plastic

Table I. Hardening/softening behavior characterized for different materials and grain boundaries

\begin{tabular}{|c|c|c|c|}
\hline Material & Maximum load/depth & Hardening/softening change & References \\
\hline $\mathrm{NiAl}$ & $1 \mathrm{mN}$ & No significant change observed & 68 \\
\hline $\mathrm{Ni}_{3} \mathrm{Al}$ & $300 \mathrm{mN}$ & No significant change observed & 69 \\
\hline Electropolished $\mathrm{Cu}$ & $90 \mu \mathrm{N}$ & $\begin{array}{c}50 \% \text { increase on one side, decrease } \\
\text { on other side. }\end{array}$ & 70 \\
\hline Bicrystalline $\mathrm{Mo}, \mathrm{Fe}-14 \% \mathrm{Si}$ & $3 \mathrm{mN}$ & $\begin{array}{l}\text { Up to } 30 \% \text { increase, for general } \\
\text { grain boundary in Fe-14\%Si; and } \\
\text { CSL(Coincident Site Lattice) } \Sigma 3 \\
\text { and CSL } \Sigma 11 \text { boundaries in Mo }\end{array}$ & 61 \\
\hline Interstitial-free steel & $500 \mu \mathrm{N}$ & $20 \%$ increase & 64 \\
\hline Nanocrystalline Ni & $100-4000 \mu \mathrm{N}$ & $15-20 \%$ increase & 71 \\
\hline Mo tricrystal & $3 \mathrm{mN}$ & $\begin{array}{l}\text { Up to } 30 \% \text { increase in hardness for } \\
\text { CSL } \Sigma 3 \text { and CSL } \Sigma 11 \text { boundary }\end{array}$ & 66 \\
\hline Inconel 690 (Ni-29Cr-9Fe) & $200 \mathrm{~nm}$ depth, $0.05 / \mathrm{s}$ strain rate & $\begin{array}{l}9 \% \text { increase for both twin and high } \\
\text { angle grain boundaries }\end{array}$ & 74 \\
\hline Bicrystalline $\mathrm{Al}$ & $200 \mathrm{~nm}$ depth, $0.05 / \mathrm{s}$ strain rate & $\begin{array}{l}\text { Up to } 15 \% \text { increase dependent on } \\
\text { strain rate of indentation, for CSL } \\
\Sigma 9 \text { tilt type boundary }\end{array}$ & 72 and 73 \\
\hline
\end{tabular}



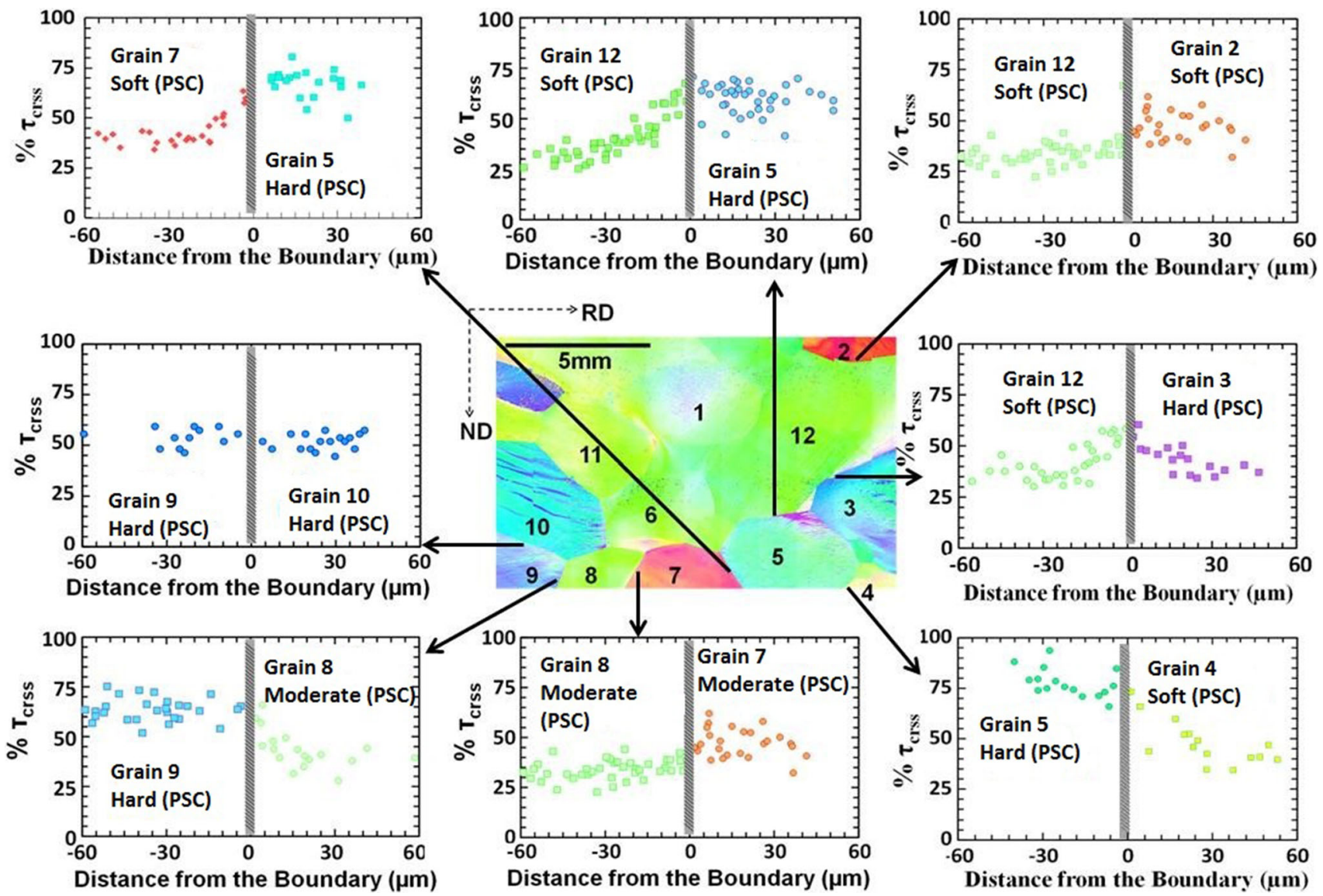

Fig. 4. Studies of grain boundary regions in plane strain compression deformed polycrystalline Al samples using spherical nanoindentation stress-strain protocols combined with electron backscattered diffraction. ${ }^{22}$ The deformed coarse-grained sample is shown with the specific grain boundary regions studied. For each grain boundary studied, the local percentage increase in critical resolved shear stress is measured as a function of the distance from the grain boundary (on both sides). Each point in these plots represents a measurement from one indentation, attesting to the high-throughput nature these protocols.

boundary in the indentation zone and $d$ is the distance of the indenter from the grain boundary. The occurrence of slip transfer in these experiments was confirmed with atomic force microscopy (AFM), which showed a pile-up of material on the other side of grain boundary (away from the indentation side). Britton and Randman ${ }^{66}$ conducted similar studies on (1) commercially pure $\mathrm{Fe}$, (2) interstitial-free steel, and (3) copper. However, GB pop-ins were only observed for commercially pure Fe for a critical $c / d$ value of 1.2 . Furthermore, contrary to earlier observations in $\mathrm{Nb},{ }^{64}$ no correlation was observed with the degree of misalignment between the active slip systems on either side of the grain boundary. Moreover, GB pop-ins were not observed in interstitial-free steel or in fcc copper, but were observed in commercial steel with interstitials. Soer et al. ${ }^{62,67}$ studied grain boundaries in $\mathrm{Fe}-14 \% \mathrm{Si}$ and $\mathrm{Mo}$ bicrystals and reported consistent GB pop-ins in $\mathrm{Fe}-14 \% \mathrm{Si}$, but not in Mo. Aifantis et al. ${ }^{65}$ also reported GB pop-ins in Fe-2.2\% Si bicrystals. Based on these observations, we can conclude that the GB pop-ins occur primarily in bcc materials (they were absent in all fcc metals studied) and demonstrate a strong correlation to the presence of interstitials. In spite of some inconsistencies between the reported observations, there is clearly tremendous potential for the indentation technique to provide new insights into the grain boundary slip transfer mechanisms.

In other studies, ${ }^{61-63,68-74}$ researchers have used a Berkovich indenter to measure the local hardness in the grain boundary region as a function of distance from the grain boundary. In general, most of these measurements show an increase in hardness in the vicinity of the grain boundaries (up to $30 \%$ ) compared to the grain interiors. Table I provides a summary of these results, where some exceptions to the general trends can be noted. For example, Soifer ${ }^{70}$ reported $50 \%$ hardness increase 
on one side of a copper grain boundary, and a decrease in hardness on the other side. Interestingly, two of the studies did not report any significant changes in the hardness across the grain boundary. ${ }^{6,69}$ As discussed earlier, hardness defined in these protocols corresponds to an indentation plastic flow stress at a finite, but nonstandardized, value of the indentation plastic strain. Consequently, one should only use the hardness measures as qualitative comparisons.

The use of the spherical indentation stress-strain protocols have produced a much more reliable and consistent set of observations. ${ }^{22,24}$ More importantly, in these studies, the objective of the spherical indentation stress-strain protocols is to lightly probe the local mechanical response in the grain boundary regions by comparing their local indentation stress-strain responses in the undeformed and deformed samples, where the deformation is imposed following standard practices in bulk forming of metals (e.g., simple compression, channel-die compression). By carefully comparing the changes in the grain boundary regions between the undeformed and the deformed conditions, it is hoped to reconstruct the roles of the different grain boundaries in the macroscopically imposed bulk plastic deformation on the sample.

A central challenge in the analyses and interpretation of the indentation stress-strain curves arises from their dependence on the crystal lattice orientation in the indentation zone. For example, any value of the measured indentation yield strength, $Y_{\text {ind }}$, is expected to be a function of both the crystal lattice orientation (with respect to the indentation direction) and the average local dislocation density in the sample. In order to separate these contributions, $Y_{\text {ind }}$ is first mapped out as a function of the crystal lattice orientation in an annealed sample (with the same chemistry as the sample of interest). ${ }^{75,76}$ In prior work, we have demonstrated that this functional dependence can be captured efficiently using generalized spherical harmonics, ${ }^{77}$ and subsequently used to predict the value of the indentation yield strength in the annealed condition for any new grain orientation of interest. Having this estimate of the indentation yield strength in the annealed condition then allows one to compute the increase in the indentation yield strength in the deformed sample (after making measurements in the deformed sample) and express this increase as a fraction of the annealed yield strength. This fraction can then be interpreted as the fractional increase in the local slip resistance at the indentation site in the deformed sample. ${ }^{75,76}$ The viability of these new protocols was recently demonstrated by Vachhani et al. $^{22}$ on a selected set of grain boundaries in a coarse-grained $\mathrm{Al}$ sample. Figure 4 summarizes the results of this study. A careful analysis of these results showed that the increased hardening in the grain boundary regions is strongly correlated to the difference in the Taylor factors of the adjoining grains. These results testify to the tremendous potential of these new high-throughput assays for systematic and rigorous studies of the mechanical role of grain boundaries (with potential extensions to phase boundaries).

\section{CONCLUSION}

This paper has reviewed and summarized the tremendous potential of indentation techniques in systematic studies of the local mechanical responses of regions at or near mesoscale interfaces and interphases. These techniques have been applied to a broad range of disparate materials systems and have been shown to be effective. In particular, the recently developed spherical indentation stressstrain protocols represent a promising new research area. Advancements in both the instrumentation and the analysis techniques have made these protocols a powerful new research tool for studying complex hierarchical materials.

\section{ACKNOWLEDGEMENTS}

SK and SM acknowledge support from NSF CMMI1333083. AR acknowledges support from the NSF IGERT-1258425 funded FLAMEL program.

\section{REFERENCES}

1. E. Baer, A. Hiltner, and H.D. Keith, Science 235, 1015 (1987).

2. A.P. Sutton and R.W. Balluffi, Interfaces in Crystalline Materials (Oxford: Clarendon Press, 1995), pp. 3-70.

3. D.P. Field and B.L. Adams, Acta Metall. Mater. 40, 1145 (1992).

4. B.L. Adams, J.W. Zhao, and D. O'Hara, Acta Metall. Mater. 38, 953 (1990).

5. K.E. Aifantis and J.R. Willis, J. Mech. Phys. Solids 53, 1047 (2005).

6. H.M. Yao and H.J. Gao, Int. J. Solids Struct. 44, 8177 (2007).

7. V. Tomar, Eng. Fract. Mech. 75, 4501 (2008).

8. T. Watanabe, Mater. Sci. Eng., A 176, 39 (1994).

9. T. Watanabe, M. Yamada, and S. Karashima, Philos. Mag. A 63, 1013 (1991).

10. R. Raj and M. Ashby, Metall. Trans. 2, 1113 (1971).

11. A.C.F. Cocks and M.F. Ashby: in Creep in Structures, ed A.R.S. Ponter and D.R. Hayhurst (Springer-Verlag Berlin, Heidelberg, NY, 1981) p. 368.

12. W. Geng, A.J. Freeman, R. Wu, C. Geller, and J. Raynolds, Phys. Rev. B 60, 7149 (1999).

13. N. Hansen, Scr. Mater. 51, 801 (2004).

14. B. Liu, D. Raabe, P. Eisenlohr, F. Roters, A. Arsenlis, and G. Hommes, Acta Mater. 59, 7125 (2011).

15. L. Lim and T. Watanabe, Acta Metall. Mater. 38, 2507 (1990).

16. G. Palumbo, E. Lehockey, and P. Lin, JOM 50, 40 (1998).

17. G. Palumbo, P. King, K. Aust, U. Erb, and P. Lichtenberger, Scr. Metall. Mater. 25, 1775 (1991).

18. M. Shimada, H. Kokawa, Z. Wang, Y. Sato, and I. Karibe, Acta Mater. 50, 2331 (2002).

19. N.J. Petch, J. Iron Steel Inst., Lond. 174, 25 (1953).

20. E.O. Hall, Proc. Phys. Soc. Lond. Sect. B 64, 747 (1951).

21. S. Sun, B.L. Adams, and W.E. King, Philos. Mag. A 80, 9 (2000).

22. S.J. Vachhani, R.D. Doherty, and S.R. Kalidindi, Int. J. Plast. 81, 87 (2016).

23. S.R. Kalidindi and S.J. Vachhani, Curr. Opin. Solid State Mater. Sci. 18, 196 (2014). 
24. S. Pathak, J. Michler, K. Wasmer, and S.R. Kalidindi, J. Mater. Sci. 47, 815 (2012).

25. A.T. DiBenedetto, Mater. Sci. Eng., A 302, 74 (2001).

26. J.-K. Kim, M.-L. Sham, and J. Wu, Compos. A 32, 607 (2001).

27. A. Eitan, K. Jiang, D. Dukes, R. Andrews, and L.S. Schadler, Chem. Mater. 15, 3198 (2003).

28. J. Zhu, A. Imam, R. Crane, K. Lozano, V.N. Khabashesku, and E.V. Barrera, Compos. Sci. Technol. 67, 1509 (2007).

29. B. Das, K. Eswar Prasad, U. Ramamurty, and C.N. Rao, Nanotechnology 20, 125705 (2009).

30. Y.X. Gan, Int. J. Mol. Sci. 10, 5115 (2009).

31. V. Cech, A. Knob, H.A. Hosein, A. Babik, P. Lepcio, F. Ondreas, and L.T. Drzal, Compos. A 58, 84 (2014).

32. S.-Y. Fu, X.-Q. Feng, B. Lauke, and Y.-W. Mai, Compos. B 39, 933 (2008).

33. S.A. Meguid and Y. Sun, Mater. Des. 25, 289 (2004).

34. V. Gertsman and S.M. Bruemmer, Acta Mater. 49, 1589 (2001).

35. Z. Shan, R.K. Mishra, S.S. Asif, O.L. Warren, and A.M. Minor, Nat. Mater. 7, 115 (2008).

36. M.D. Uchic, D.M. Dimiduk, J.N. Florando, and W.D. Nix, Science 305, 986 (2004).

37. A. Kunz, S. Pathak, and J.R. Greer, Acta Mater. 59, 4416 (2011).

38. V. Rao, P. Herrera-Franco, A. Ozzello, and L. Drzal, J. Adhes. 34, 65 (1991).

39. W. Hao, C. Tang, Y. Yuan, X. Yao, and Y. Ma, Polym. Test. 41, 239 (2015)

40. R.F. Gibson, Compos. Sci. Technol. 105, 51 (2014).

41. S. Pathak and S.R. Kalidindi, Mater. Sci. Eng., $R$ 91, 1 (2015).

42. S. Pathak, D. Stojakovic, R. Doherty, and S.R. Kalidindi, J. Mater. Res. 24, 1142 (2009).

43. P. Herrera-Franco and L. Drzal, Composites 23, 2 (1992).

44. A. Hodzic, Z.H. Stachurski, and J.K. Kim, Polymer 41, 6895 (2000).

45. A. Hodzic, J.K. Kim, and Z.H. Stachurski, Polymer 42, 5701 (2001).

46. A. Hodzic, J.K. Kim, A.E. Lowe, and Z.H. Stachurski, Compos. Sci. Technol. 64, 2185 (2004).

47. A. Hodzic, S. Kalyanasundaram, J.K. Kim, A.E. Lowe, and Z.H. Stachurski, Micron 32, 765 (2001).

48. H. Wang, Y. Bai, S. Liu, J. Wu, and C.P. Wong, Acta Mater. 50, 2369 (2002)

49. T.J. Young, L.E. Crocker, W.R. Broughton, S.L. Ogin, and P.A. Smith, Compos. A 50, 39 (2013).

50. G. Mallikarjunachari and P. Ghosh, Polymer 90, 53 (2016).

51. S. Pathak, S.J. Vachhani, K.J. Jepsen, H.M. Goldman, and S.R. Kalidindi, J. Mech. Behav. Biomed. Mater. 13, 102 (2012).
52. S. Pathak, J.G. Swadener, S.R. Kalidindi, H. Courtland, K.J. Jepsen, and H.M. Goldman, J. Mech. Behav. Biomed. Mater. 4, 34 (2011).

53. S. Pathak, Z.G. Cambaz, S.R. Kalidindi, J.G. Swadener, and Y. Gogotsi, Carbon 47, 1969 (2009).

54. S.R. Kalidindi and S. Pathak, Acta Mater. 56, 3523 (2008).

55. S. Pathak, J. Shaffer, and S.R. Kalidindi, Scr. Mater. 60, 439 (2009).

56. H. Hertz, Miscellaneous Papers (London: MacMillan, 1896), pp. $146-163$.

57. M. Desaeger and I. Verpoest, Compos. Sci. Technol. 48, 215 (1993).

58. H. Ho and L.T. Drzal, Compos. A 27, 961 (1996).

59. S. Pathak, J.L. Riesterer, S.R. Kalidindi, and J. Michler, Appl. Phys. Lett. 105, 161913 (2014).

60. T. Ohmura, K. Tsuzaki, and F.X. Yin, Mater. Trans. 46, 2026 (2005)

61. T. Ohmura and K. Tsuzaki, J. Phys. D Appl. Phys. 41, 074015 (2008).

62. W.A. Soer, K.E. Aifantis, and J.T.M. De Hosson, Acta Mater. 53, 4665 (2005).

63. T. Eliash, M. Kazakevich, V.N. Semenov, and E. Rabkin, Acta Mater. 56, 5640 (2008).

64. M.G. Wang and A.H.W. Ngan, J. Mater. Res. 19, 2478 (2004).

65. K.E. Aifantis, W.A. Soer, J.T.M. De Hosson, and J.R. Willis, Acta Mater. 54, 5077 (2006).

66. T.B. Britton, D. Randman, and A.J. Wilkinson, J. Mater. Res. 24, 607 (2009).

67. W.A. Soer and J.T.M. De Hosson, Mater. Lett. 59, 3192 (2005).

68. M. Göken, M. Kempf, M. Bordenet, and H. Vehoff, Surf. Interface Anal. 27, 302 (1999).

69. P.C. Wo and A.H.W. Ngan, J. Mater. Res. 19, 189 (2004).

70. Y.M. Soifer, A. Verdyan, M. Kazakevich, and E. Rabkin, Scr. Mater. 47, 799 (2002).

71. B. Yang and H. Vehoff, Acta Mater. 55, 849 (2007).

72. G.Z. Voyiadjis and C. Zhang, Mater. Sci. Eng., A 621, 218 (2015).

73. C. Zhang and G.Z. Voyiadjis, Mater. Sci. Eng., A 659, 55 (2016).

74. F.W. Herbert, B. Yildiz, and K.J. Van Vliet: MRS Proceedings 1297, 187 (2011).

75. S.J. Vachhani and S.R. Kalidindi, Acta Mater. 90, 27 (2015).

76. S. Pathak, D. Stojakovic, and S.R. Kalidindi, Acta Mater. 57, 3020 (2009).

77. H. Bunge, Texture analysis in materials science (London: Butterworths, 1982), pp. 47-118. 